\title{
DNA-doxorubicin interaction: New insights and peculiarities
}

\author{
E. F. Silva | R. F. Bazoni \\ Laboratório de Física Biológica, \\ Departamento de Física, Universidade \\ Federal de Viçosa, Viçosa, Minas \\ Gerais, Brazil
}

\section{Correspondence}

M. S. Rocha Laboratório de Física Biológica, Departamento de Física, Universidade Federal de Viçosa, Viçosa, Minas Gerais, Brazil. Email: marcios.rocha@ufv.br

\section{Funding Information}

Brazilian agencies: Fundação de Amparo à Pesquisa do Estado de Minas Gerais (FAPEMIG), Conselho Nacional de Desenvolvimento Científico e Tecnológico (CNPq) and Coordenação de Aperfeiçoamento de Pessoal de Nível Superior (CAPES).

\begin{abstract}
We have investigated the interaction of the DNA molecule with the anticancer drug doxorubicin (doxo) by using three different experimental techniques: single molecule stretching, single molecule imaging, and dynamic light scattering. Such techniques allowed us to get new insights on the mechanical behavior of the DNA-doxo complexes as well as on the physical chemistry of the interaction. First, the contour length data obtained from single molecule stretching were used to extract the physicochemical parameters of the DNA-doxo interaction under different buffer conditions. This analysis has proven that the physical chemistry of such interaction can be modulated by changing the ionic strength of the surrounding buffer. In particular we have found that at low ionc strengths doxo interacts with DNA by simple intercalation (no aggregation) and/or by forming bound dimers. For high ionic strengths, otherwise, doxo-doxo self-association is enhanced, giving rise to the formation of bound doxo aggregates composed by 3 to 4 molecules along the doublehelix. On the other hand, the results obtained for the persistence length of the DNAdoxo complexes is strongly force-dependent, presenting different behaviors when measured with stretching or non-stretching techniques.
\end{abstract}

KE Y W O R D S

doxorubicin, single molecule, physical chemistry, mechanical properties

\section{1 | INTRODUCTION}

Doxorubicin (doxo) is a well-known chemotherapeutic compound used to treat various cancers such as some types of leukemias, sarcomas, lymphomas, myelomas, neuroblastomas, as well as cancers in the breast, head, ovary, pancreas, prostate, stomach, liver, lung, and others. Along with the related compounds daunomycin, mitoxantrone and idarubicin, they constitute the class of the anthracycline antibiotics, a group of intercalators largely employed in chemotherapies. ${ }^{[1]}$

In this work we have performed a robust characterization of the DNA-doxo interaction at single molecule level. We have used optical tweezers (OT) in the low-force entropic regime to stretch the DNA-doxo complexes in order to measure the changes on the basic mechanical properties (persistence and contour lengths) of such complexes as a function of the drug concentration in the sample. In addition, we have also performed single molecule imaging of the DNA-doxo complexes by using atomic force microscopy (AFM). In these experiments, the DNA-doxo complexes deposited in a mica substrate were imaged and the same mechanical properties were obtained directly from the statistical analysis of the conformation of the complexes. Thus, the results obtained from single molecule stretching and imaging could be directly compared, bringing new insights on the mechanics of the DNA complexes formed with intercalators. In particular, we have found that the behavior of the persistence length of the DNA-doxo complexes obtained from these two techniques is very different, being strongly force-dependent.

On the other hand, an important aspect concerning the molecular basis of the chemotherapies is the physical chemistry of the DNA-drugs interactions, especially the information about the possible types of binding modes, drug affinity, selectivity, cooperativity, etc. Some of such information is currently known for the DNA-doxo interaction, albeit some aspects remain unclear. In particular, it is well established that intercalation is the main mode of interaction, ${ }^{[2-5]}$ 
although some authors report the possibility of groove binding at AT-rich regions. ${ }^{[3]}$ Most of the available information was determined from ensemble-averaging techniques such as circular dichroism, fluorescence and infrared spectroscopy, microcalorimetry, etc., ${ }^{[3-5]}$ and very few authors have used single molecule approaches to investigate the DNA-doxo interaction. ${ }^{[2,6]}$ Single molecule techniques such as optical and magnetic tweezers, AFM and fluorescence-based techniques usually allow one to obtain high-resolution information about DNA-ligand binding, revealing intrinsic details of the interaction that are otherwise inaccessible. ${ }^{[7-20]}$

In the present work, besides the new mechanical insights on the DNA-doxo interaction, our single molecule measurements have allowed us to: (a) infer that different binding mechanisms can occur depending on the ionic strength of the buffer solution; and (b) determine the physicochemical parameters of the DNA-doxo interaction under different buffer conditions. In particular, we have found that in general doxo binds to the DNA molecule forming aggregates of a few molecules, which remain partially intercalated. The size of these aggregates, as well as the other binding parameters, can be controlled by changing the ionic strength of the buffer solution. In other words, the physical chemistry of the DNAdoxo interaction can be modulated by changing the surrounding buffer.

Finally, the data obtained from the single molecule techniques were compared to results obtained by an ensembleaveraging technique: dynamic light scattering (DLS). We have used DLS in order to evaluate the behavior of the hydrodynamic radius of DNA-doxo complexes as a function of drug concentration. This quantity can be qualitatively compared to the radius of gyration calculated from the persistence and contour lengths obtained from OT and $\mathrm{AFM},{ }^{[21,22]}$ thus connecting the results of all experiments performed here. All measurements and analysis performed here can be extended for other types of intercalators, thus providing new insights on the DNA interactions with this class of drugs.

\section{2 | MATERIALS AND METHODS}

\section{$2.1 \mid$ Optical tweezers (OT)}

In OT experiments the samples consist of $\lambda$-DNA molecules (New England Biolabs) end-labeled with biotin attached by one end to a streptavidin-coated bead of $3 \mu \mathrm{m}$ diameter (Bangs Labs) and by the other end to a streptavidin-coated coverslip (Xenopore Corp.). The sample chamber consists of an o-ring glued in the coverslip surface. In order to evaluate the effects of the ionic strength in the DNA-doxo interaction, we have performed the measurements in two different buf- fers, using a $10 \mathrm{mM}$ Tris- $\mathrm{HCl}$ buffer with $\mathrm{pH}=7.4$ without $\mathrm{NaCl}$ and also a Phosphate Buffered Saline (PBS) buffer with $\mathrm{pH}=7.4$ and $[\mathrm{NaCl}]=140 \mathrm{mM}$. Although the composition of the buffers is not exactly the same, the relevant parameter here is the difference between the ionic strengths ( $>$ one order of magnitude), because the self-association of the anthracyclinic compounds strongly depends on this parameter ${ }^{[23,24]}$. The doxo concentration in the sample was changed during the experiments by using micropipettes to exchange the buffer solution. The typical DNA concentration used in all OT experiments was $2.4 \mu \mathrm{M}$ in base-pairs.

The optical tweezers consist of a $1064 \mathrm{~nm}$ ytterbiumdoped fiber laser with a maximum output power of $5.8 \mathrm{~W}$ (IPG Photonics) mounted on a Nikon Ti-S inverted microscope with a $100 \times$ N.A. 1.4 objective. The DNA molecules are stretched by moving the microscope stage and consequently the coverslip with controlled velocity by using a piezoelectric device (PINano P-545, Physik Instrumente).

We start the experiment with only bare DNA molecules in the sample. We choose and test one of them by measuring 5 to 7 stretching curves, obtaining the mean values of the persistence and contour lengths for the bare DNA. These parameters were obtained by fitting the experimental force $\times$ extension curves measured in the low-force entropic regime $(F<5 \mathrm{pN})$ to the Marko-Siggia WormLike Chain (WLC) expression. ${ }^{[25]}$ The average results obtained for the bare DNA in both buffers are $A_{0}=(45 \pm 3) \mathrm{nm}$ and $L_{0}=(16.5 \pm 1) \mu \mathrm{m}$, which are within the expected values for the bare $\lambda$-DNA. Next we change the surrounding buffer solution, introducing the drug at a certain chosen concentration. We wait about $\sim 20$ minutes for drug equilibration, and then repeat the stretching experiments, performing 5 to 7 measurements and thus obtaining the average values and the error bars of the mechanical properties for each drug concentration. Finally, the entire experiments is repeated with other DNA molecules, in order to evaluate the variability of the mechanical parameters over different DNAs. The results reported here for the persistence and contour lengths correspond to an average over 4 to 6 different DNA-doxo complexes. All the error bars reported are the standard error of the mean (sem) calculated from the set of stretching experiments for each drug concentration.

All the details about the WLC fittings and some exemplifying figures can be found in the Supporting Information. The details about the OT sample preparation procedure and about the optical tweezers setup were previously described. ${ }^{[26,27]}$

\section{$2.2 \mid$ Atomic force microscopy (AFM)}

The samples here consist of $3 \mathrm{kbp}$ DNA molecules (Thermo Scientific) in the same Tris-HCl buffer used in OT 
experiments, except by the addition of $10 \mathrm{mM}$ of $\mathrm{MgCl}_{2}$, which is needed in order to deposit the DNA molecules in mica substrates. The mixture was allowed to equilibrate for $\sim 20 \mathrm{~min}$. An aliquot of $20 \mu \mathrm{l}$ was deposited on the substrate and completely dried out with nitrogen at ambient temperature $\left(\sim 25^{\circ} \mathrm{C}\right)$. To compare the morphologies observed with the results obtained in the OT experiments, we have used similar ratios of drug concentration by DNA base-pair concentration. The $3 \mathrm{kbp}$ DNA was used to allow the visualization of various different molecules in the scanned images and to avoid relevant volume-exclusion effects present for the $\lambda$-DNA, due to its long contour length $(48.5 \mathrm{kbp}) .{ }^{[28]}$ The PBS buffer could not be used here because the high $\mathrm{NaCl}$ concentration disturbs the DNA adsorption on the substrates.

The mica substrates were scanned with the AFM (model NTEGRA, NT-MDT-Russia) operating in the conventional semicontact mode at a scan rate in the range of $1.5-3.0 \mathrm{~Hz}$. We have used NanoWorld tips with radius equal to $8 \mathrm{~nm}$ and a force constant on the order of a few N/m. The experiments were performed in air, at ambient temperature and with humidity $\sim 20 \%-30 \%$. This experimental procedure has been shown suitable to visualize the deposited DNA and DNA-drug complexes in a reproducible and reliable way. ${ }^{\text {[27] }}$ In the Supporting Information we show some representative AFM images of the DNA-doxo complexes deposited in mica substrates.

To analyze the images of the deposited DNA-doxo complexes, we have determined the mean contour and persistence lengths and the error bars (sem) for each drug concentration ( $\sim 70$ different molecules for each concentration). The analysis was performed following the procedure of Rivetti et al. ${ }^{[28]}$ Basically, we measure the contour length $L$ and the mean-squared end-to-end distance $\left\langle R^{2}\right\rangle$ of the polymer chain directly from the images. The persistence length $A$ can then be determined with the equation

$$
<R^{2}>=4 A L\left[1-\frac{2 A}{L}\left(1-e^{-\frac{L}{2 A}}\right)\right],
$$

which is valid for 2D worm-like chains. ${ }^{[28]}$

\section{3 | Dynamic light scattering (DLS)}

All DLS measurements were performed in the apparatus ZetaSizer Nano-S (Malvern Instruments Ltd.) with a low volume quartz cuvette (ZEN2112, Hellma Analytics). The samples here consist of $3 \mathrm{kbp}$ DNA molecules (Thermo Scientific) in the same PBS buffer used in the OT experiments ( $\lambda$-DNA is difficult to be used in DLS due to the long contour length). The DNA molecules are equilibrated with a certain doxo concentration directly in the cuvette used. The DNA concentration used in all DLS experiments was 4.8 $\mu \mathrm{M}$ of base-pairs. This concentration is sufficiently low to avoid entanglements and relevant interactions between different DNA molecules ${ }^{[29]}$. We show the results of the experiments here only in PBS because the results obtained by this technique using our two different buffers are indistinguishable within the experimental error bars.

We have measured seven different samples with increasing concentrations of doxo, in order to investigate the effect of the ligand on the effective size of the DNA molecule, measured here by the hydrodynamics radius $R_{H}$, which is obtained directly from the intensity autocorrelation functions of the scattered light (representative raw data can be found in the Supporting Information). For each doxo concentration, we have performed $\sim 70$ measurements of 15 seconds each, in order to obtain the mean results and the error bars (sem). More experimental details can be found in Refs. 21 and 22.

\section{3 | RESULTS AND DISCUSSION}

\subsection{The contour length can be used to deduce the physical chemistry of the interaction}

Optical tweezers experiments with DNA-doxo complexes were performed in order to determine the changes on the basic mechanical properties (persistence and contour lengths) as a function of the drug concentration in the sample. Recently, we have developed a methodology to extract the physical chemistry of the interaction from these mechanical parameters, such that a robust and nearly complete characterization can be performed with a very reduced number of experimental techniques. ${ }^{[18,22,26,27,30-32]}$

In Figure 1 we show the relative increase of the contour length $\Theta=\left(L-L_{0}\right) / L_{0}$ of the DNA-doxo complexes as a function of doxo total concentration in the sample normalized by the DNA base-pair concentration $\left(C_{\mathrm{T}} / C_{\mathrm{bp}}\right)$, obtained in Tris- $\mathrm{HCl}$ (blue squares) as well as in the PBS buffer (black circles). Observe that in both situations the contour length increases monotonically from the bare $\lambda$-DNA value $(\Theta=0)$ up to a saturation value $(\Theta \sim 0.28$ in Tris- $\mathrm{HCl}$ and $\Theta \sim 0.21$ in PBS). Since intercalative binding is directly related to the increase of the contour length, ${ }^{[18]}$ these data suggest that doxo intercalation into DNA is favored in the Tris- $\mathrm{HCl}$ buffer, which has lower ionic strength $([\mathrm{NaCl}]=0)$.

In addition, observe that the data obtained in the Tris$\mathrm{HCl}$ buffer presents the typical shape observed for most intercalators. $^{[21,22,33-35]}$ The data obtained in the PBS buffer, on the other hand, exhibits a slightly sigmoidal shape which indicates that another binding mode may exist besides intercalation or/and significant cooperativity can exist between the ligand molecules. ${ }^{[18]}$

A recent work from Arnaiz et al. demonstrated that the doxo molecules can also bind outside the DNA double-helix, 


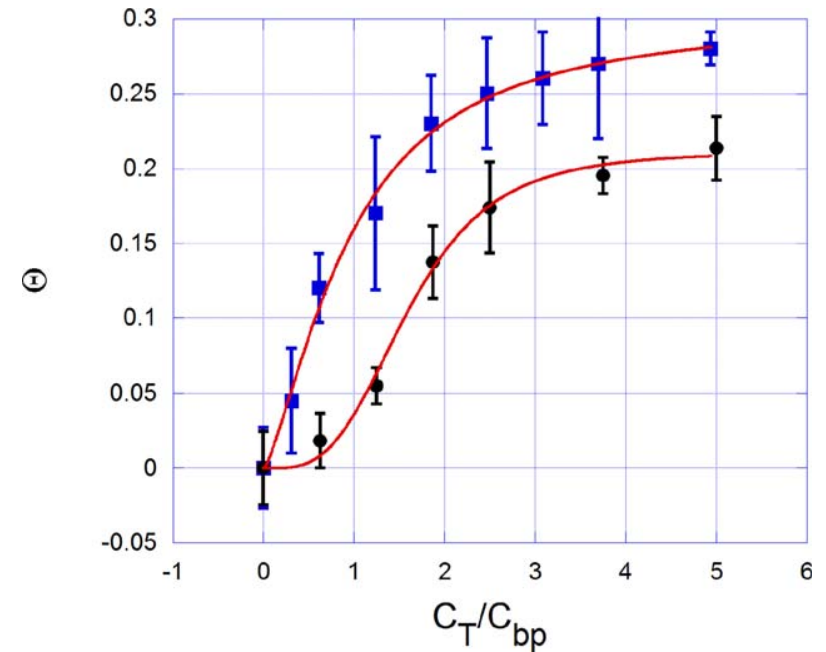

FIGURE 1 Relative increase of the contour length $\Theta=(L$ $\left.L_{0}\right) / L_{0}$ as a function of doxo concentration in the sample normalized by the DNA base-pair concentration $\left(C_{\mathrm{T}} / C_{\mathrm{bp}}\right)$, obtained with OT both in Tris- $\mathrm{HCl}$ (blue squares) as well as in the PBS buffer (black circles). The solid lines are fittings to the equation $\Theta=\gamma r$ expressing $r$ as a Hill binding isotherm

interacting with a previously intercalated doxo molecule. ${ }^{[3]}$ Such result is related to the fact that many anthracycline antibiotics have a tendency to self-associate in solution, ${ }^{[23,24]}$ and such association is strengthened in high salt concentrations, ${ }^{[23]}$ probably due to the screening of the doxo-doxo electrostatic repulsion. A similar sigmoidal behavior of the contour length as a function of drug concentration was previously obtained by us for the closely related drug daunomycin under the same experimental conditions. ${ }^{[33]}$

In order to understand the effect of the ionic strength on the doxo binding and to extract the physicochemical parameters of the interaction, we can fit the data of Figure 1 to a convenient binding isotherm. ${ }^{[18]}$ It is well established for intercalators that $\Theta=\gamma r$, where $\gamma$ is a constant typically $\sim 1$ (the ratio between the extension elongated per ligand and the distance between two consecutive base-pairs) and $r$ is the fraction of bound ligand per DNA base-pair, whose saturation value is $r_{\max }{ }^{[21,22,27,33]}$ To perform the fitting, the bound ligand fraction $r$ can be expressed by a convenient binding isotherm that captures the physical chemistry of the interaction. ${ }^{[18]}$

For molecules which interact to the DNA only by simple intercalation, there are some options to be chosen as the binding isotherm in the fitting process: the McGhee - von Hippel neighbor exclusion model ${ }^{[36]}$ or, alternatively, the Hill model. ${ }^{[18]}$ While both isotherms explain well the monotonic increase of $\Theta$ observed in the Tris- $\mathrm{HCl}$ buffer, the classic McGhee - von Hippel binding isotherm (without cooperativity) cannot account for the sigmoidal behavior of
$\Theta$ obtained in the PBS buffer (see Figure 1). Therefore, in order to perform a robust comparison between the data obtained in the two different buffers, we have chosen the Hill model to fit both data, thus avoiding systematic errors related to the use of different binding isotherms. Such model is capable to account for ligand aggregation along the double helix, ${ }^{[30,32]}$ a feature usually observed for anthracyclines due to their self-association. ${ }^{[23,24]}$ The Hill binding isotherm reads

$$
r=\frac{r_{\max }\left(K_{i} C_{f}\right)^{n}}{1+\left(K_{i} C_{f}\right)^{n}} .
$$

where $K_{i}$ is the equilibrium binding association constant, $n$ is the Hill exponent - a cooperativity parameter which is a lower bound for the number of cooperating ligand molecules involved in the reaction, ${ }^{[27,30]}$ and $C_{f}$ is the free (not bound) ligand concentration in solution.

The fittings were performed using the numerical approach detailed described in Ref. 18. To reduce the number of adjustable parameters, we have fixed $\gamma=1$, the expected value for simple monointercalators such as the anthracyclines. ${ }^{[18,22]}$ The fittings are shown in Figure 1 (solid lines). From these fittings, we extract the physicochemical parameters of the interaction. For the Tris-HCl buffer, $K_{i}=(5.3 \pm 1.3) \times 10^{5} \mathrm{M}^{-1}$, $n=1.4 \pm 0.4$, and $r_{\max }=0.30 \pm 0.04$. For the PBS buffer, on the other hand, $K_{i}=(2.7 \pm 0.3) \times 10^{5} \mathrm{M}^{-1}, n=3.6 \pm 0.7$, and $r_{\text {max }}=0.21 \pm 0.02$.

The values obtained for the equilibrium association constants are on the same order of magnitude of the results obtained for other anthracyclines. ${ }^{[35,37]}$ Observe that $K_{i}$ is approximately twice higher for the interaction in the Tris$\mathrm{HCl}$ buffer. Such result is related to the fact that a lower salt concentration strengths the electrostatic interaction between the negative phosphate groups of the DNA backbone and the doxo molecules (which are monocationic), thus enhancing intercalation.

The Hill exponent allows us to estimate the size of the doxo aggregates bound along the double-helix. ${ }^{[18,30,32]}$ The value $n=1.4 \pm 0.4$ obtained in the Tris- $\mathrm{HCl}$ buffer indicates that doxo interacts with DNA in this buffer by simple intercalation (no aggregation) and/or by forming bound dimers. Such conclusion is compatible to the results found by Arnaiz et al., who have achieved the same conclusion using microcalorimetry and various spectroscopies in a buffer with a very low ionic strength $([\mathrm{NaCl}]=2.5 \mathrm{mM}) .{ }^{[3]}$ These authors have proposed that the dimers are composed by a partially intercalated doxo molecule which interacts and aggregate with another doxo molecule that remains outside the doublehelix. ${ }^{[3]}$ We think that this is the only possible picture here, since two or more aggregated doxo molecules cannot intercalate together. On the other hand, the value $n=3.6 \pm 0.7$ obtained in the PBS buffer indicates that, in this situation, doxo aggregates bound to DNA are composed by 3 to 4 


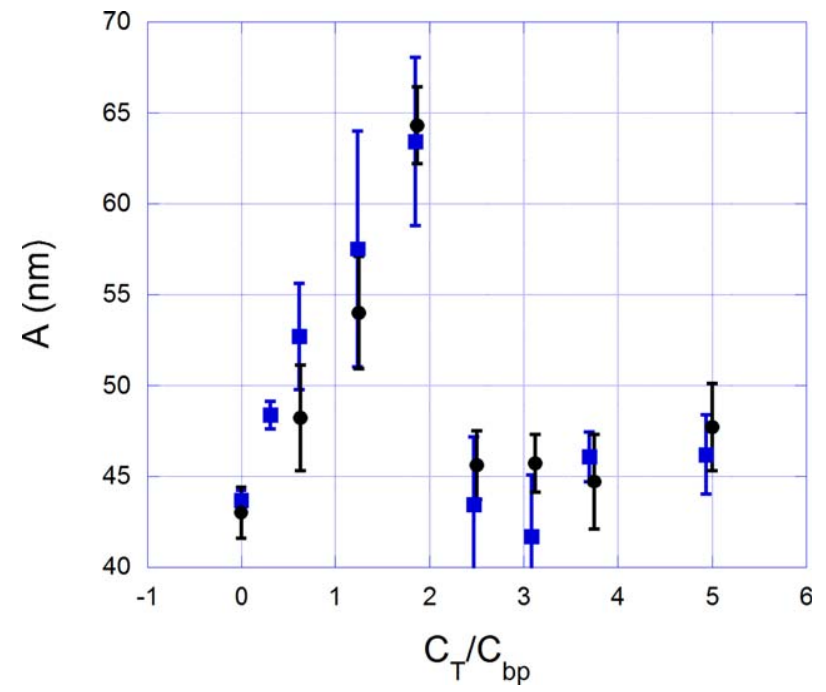

FIGURE 2 Persistence length $A$ of DNA-doxo complexes as a function of drug total concentration in the sample normalized by the DNA base-pair concentration $\left(C_{\mathrm{T}} / C_{\mathrm{bp}}\right)$, obtained with OT both in the Tris- $\mathrm{HCl}$ (blue squares) as well as in the PBS buffer (black circles). Observe that $A$ initially increases from the bare DNA value ( $\sim 3 \mathrm{~nm}$ ) up to a maximum value ( $\sim 64 \mathrm{~nm}$ ) reached at $C_{\mathrm{T}} / C_{\mathrm{bp}} \sim 1.9$, and then abruptly decreases to around $\sim 45 \mathrm{~nm}$ and remains constant within the error bars

doxo molecules in average. Here, again, only one molecule should be partially intercalated, while the others stay outside the double-helix. This result is compatible to the one found by Chaires et al. for the related anthracycline daunomycin. ${ }^{[23]}$ These authors have found that high order daunomycin aggregation occurs under $[\mathrm{NaCl}]=185 \mathrm{mM}$, showing that a model with aggregates composed of four molecules can in principle explain their binding data. ${ }^{[23]}$ In fact, the Debye length is about $\sim 4$ times higher in the Tris- $\mathrm{HCl}$ buffer relative to the PBS buffer, and thus a much higher doxo aggregation is expected in the PBS buffer due to the screening of the electrostatic repulsion between the monocationic doxo molecules. A similar aggregation behavior was recently found for the cyclodextrin molecule when interacting with DNA, although in this case the electrostatic repulsion was modulated by changing the surface charge of the ligand. ${ }^{[30,32]}$ Other works can be found in the literature reporting ligand aggregation along the DNA doublehelix. ${ }^{[26,31,38,39]}$

Finally, the bound ligand fraction at saturation $\left(r_{\max }\right)$ is considerable higher in the Tris-HCl buffer $(\sim 0.3)$ than in the PBS buffer $(\sim 0.21)$. This result is due to the fact that a doxo trimer/tetramer occupy more space along the double-helix than a dimer or a single doxo molecule, which reduces the effective number of available intercalation sites in the PBS buffer. In fact, the effective binding site size can be estimated as $1 / r_{\max },{ }^{[18]}$ being $\sim 3.3$ base-pairs in the Tris-HCl buffer and $\sim 4.8$ base-pairs in the PBS buffer. Observe that only intercalated doxo molecules (and not the rest of the aggregate which remains outside the double helix ${ }^{[3]}$ ) contribute to the increase of the contour length. Thus, we really expect a lower increase in the contour length under higher ionic strengths.

\subsection{The persistence length is strongly force-dependent}

In Figure 2 we show the behavior of the persistence length $A$ as a function of doxo total concentration in the sample $\left(C_{\mathrm{T}}\right)$ normalized by the DNA base-pair concentration $\left(C_{\mathrm{bp}}\right)$, obtained with OT. As mentioned before, the experiments were performed both in the Tris- $\mathrm{HCl}$ (blue squares) as well as in the PBS buffer (black circles), and the behavior found in the two situations is the same within the error bars. Observe in fact that in both situations the persistence length initially increases from the bare DNA value $(\sim 43 \mathrm{~nm})$ up to a maximum value $(\sim 64 \mathrm{~nm})$ reached at $C_{\mathrm{T}} / C_{\mathrm{bp}} \sim 1.9$, and then abruptly decreases to around $\sim 45 \mathrm{~nm}$ and remains constant within the error bars at least for the concentration range studied here.

Such behavior of the persistence length appears to be a general property of DNA-intercalator complexes when stretched under the force regime of $F<5 \mathrm{pN}^{\left[{ }^{[40]}\right.}$ In fact, the same qualitative behavior was previously verified under our experimental

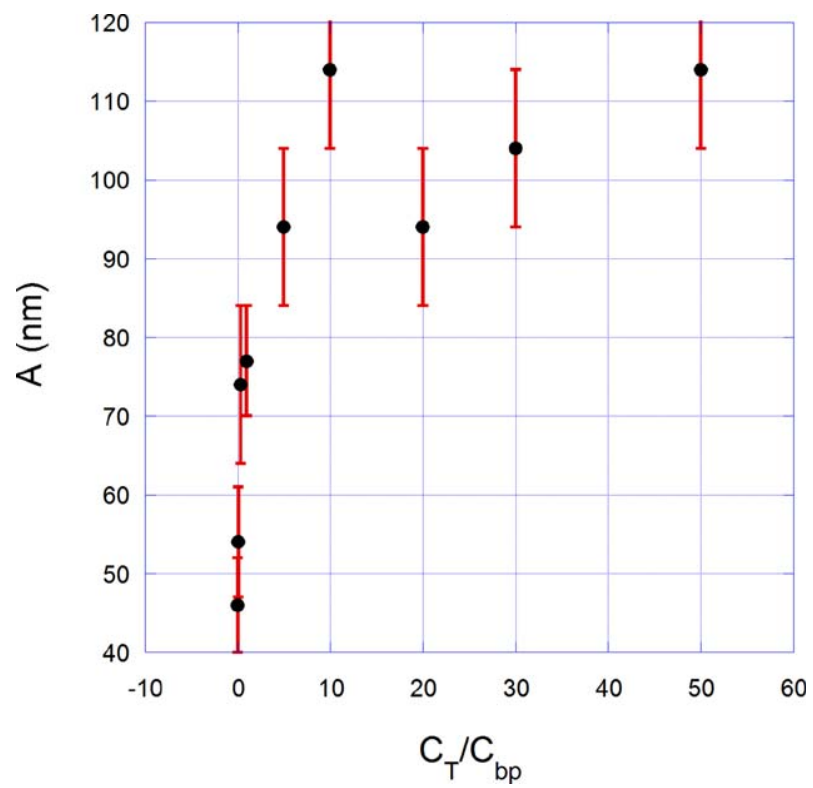

FIGURE 3 Persistence length $A$ of DNA-doxo complexes as a function of drug total concentration in the sample normalized by the DNA base-pair concentration $C_{\mathrm{T}} / C_{\mathrm{bp}}$, obtained by AFM. Observe that $A$ monotonically increases from the bare DNA value until the saturation value of $\sim 110 \mathrm{~nm}$, differing drastically from the behavior obtained in single molecule stretching experiments performed by optical tweezers 
conditions for the intercalators ethidium bromide ${ }^{[22,33]}$ daunomycin, ${ }^{[33]}$ psoralen, ${ }^{[34]}$ diaminobenzidine ${ }^{[21]}$ and for the bisintercalator gelred. ${ }^{[22]}$ As detailed discussed in Ref. 40; however, such behavior depends strongly on some experimental features, especially on the force regime used to stretch the DNA-intercalator complexes.

In fact, in Figure 3 we show the behavior of the persistence length obtained from the AFM experiments for the DNA-doxo complexes under the experimental conditions previously discussed. Observe that here the persistence length increases monotonically from the bare DNA value until the saturation value of $\sim 110 \mathrm{~nm}$, differing drastically from the behavior obtained in the stretching experiments. In order to guarantee that no abrupt decrease on this parameter occurs in the AFM experiments, we have used doxo concentrations as high as $C_{\mathrm{T}} / C_{\mathrm{bp}} \sim 50$, i.e., 10 times higher then the saturation value found in OT experiments.

As mentioned before, such apparent discrepancy between the OT and AFM data can be understood on the basis of the extensive discussion found in Ref. 40. Basically, the persistence length of DNA-intercalator complexes is in general force-dependent. The general tendency of intercalators is to increase the DNA persistence length as a result of the various local structures formed along the double-helix upon drug binding, which are stabilized by hydrophobic stacking interactions between the drug molecules and the adjacent basepairs. Nevertheless, depending on the drug concentration and/or the force regime used to perform the experiments with single molecule stretching techniques, a partial melting of the double-helix structure can occur due the stretching forces applied on the highly distorted double-helix structure of the DNA-intercalator complexes. It is well established that intercalators locally unwind the double-helix upon binding, exerting a torque that distorts the hydrogen bonds around the intercalation site. It was previously demonstrated that this kind of structural change, when under tension, can melt the double-helix locally, ${ }^{[41]}$ forming denaturation bubbles which induce a decrease on the effective persistence length. ${ }^{[42,43]}$ In the AFM experiments, there are no applied external forces, such that one should expect that the persistence length increases and saturates as the drug binds, which is exactly the behavior shown in Figure 3.

As discussed in Ref. 40, besides the partial melting assumption, an important issue related to the above results is the fact that the external force applied to stretch the DNAdrug complexes can change the chemical equilibrium between the drug and the DNA molecule. In fact, Vladescu et al. have shown that binding parameters such as the equilibrium association constant and the binding site size depend on the force applied on the DNA-drug complexes. In particular, the equilibrium constant increases exponentially as a

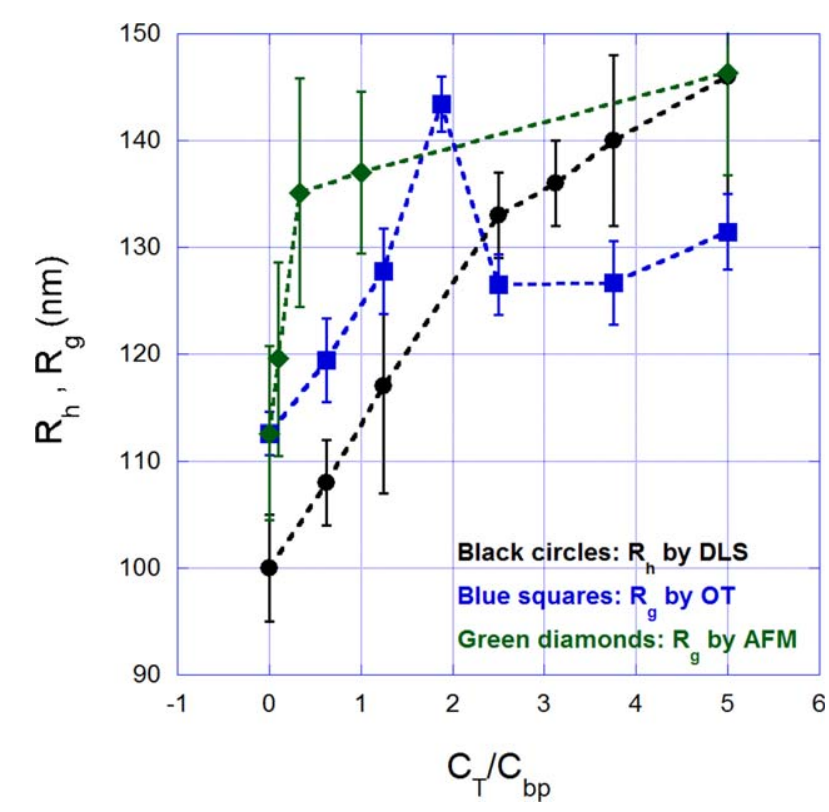

FIGURE 4 Black circles: hydrodynamic radius $R_{\mathrm{h}}$ obtained from the DLS experiments as a function of the normalized drug concentration in the sample $\left(C_{\mathrm{T}} / C_{\mathrm{bp}}\right)$. For comparison purposes, it is also shown the radius of gyration $R_{\mathrm{g}}$ of such complexes obtained both from optical tweezers (OT) (PBS buffer) (blue squares) and AFM (green diamonds) data. The dashed lines are guides to the eyes

function of the applied force ${ }^{[44]}$ Since this constant is closely linked to the concentration of bound drug, it is expected that the mechanical properties of the DNA-drug complexes are in fact force-dependent. Another work that evidences such conclusion was recently performed by Camunas-Soler et al. ${ }^{[16]}$ Nevertheless, it is difficult to explain the non-monotonic behavior of the persistence length only with this assumption, since one or more binding parameters should abruptly change their values at the "critical concentration" where the persistence length invert its behavior. In addition, since here we have used only very small forces to stretch the DNAdoxo complexes $(F<5 \mathrm{pN})$, the changes on the binding parameters are not relevant. Thus, we believe that only a structural change such as partial melting can explain the abrupt decrease observed in the persistence length measured by OT.

\subsection{DLS experiments corroborates with the partial melting proposal}

In order to put into test the above discussion about the partial melting, a third experimental technique was used here. DLS was chosen because it is completely different from OT and AFM, being an ensemble-averaging technique which gives the mean behavior of a very high number of molecules. 
In Figure 4 (black circles) we show the behavior of the hydrodynamic radius $R_{\mathrm{h}}$ obtained from the DLS experiments as a function of the normalized drug concentration in the sample $\left(C_{\mathrm{T}} / C_{\mathrm{bp}}\right)$ (the dashed lines in this figure are only guides to the eyes). Observe that $R_{\mathrm{h}}$ increases monotonically as a function of the drug concentration, which indicates an increase on the effective size of the DNA-doxo complexes.

For comparison purposes, we also plot in Figure 4 the radius of gyration $R_{g}$ of the DNA-doxo complexes obtained both from the OT (PBS buffer) (blue squares) and AFM (green diamonds) data. The radius of gyration was obtained from the corresponding persistence and contour lengths data by using the relation ${ }^{[45]}$

$$
R_{g}=\sqrt{\frac{1}{3} A L\left(1-\frac{3 A}{L}+\ldots\right)},
$$

where $A$ is the persistence length and $L$ is the contour length of the DNA-doxo complexes. This equation predicts that $R_{\mathrm{g}}$ increases with both $A$ and $L$, as expected intuitively. In the case of OT experiments, which use $\lambda$-DNA (48.500 bp), the contour lengths were multiplied by the factor $3 / 48.5$ in order to perform the comparison with the $3000 \mathrm{bp}$ DNA used in the DLS and AFM experiments.

Although $R_{\mathrm{h}}$ and $R_{\mathrm{g}}$ cannot be quantitatively compared ( $R_{\mathrm{h}}$ represents the radius of the equivalent sphere with the same diffusion coefficient and $R_{\mathrm{g}}$ is directly derived from pure mechanical parameters), they should exhibit the same qualitative behavior as a function of drug concentration, since both quantities are related to the effective size of the DNA-drug complexes. ${ }^{[21]}$ Nevertheless, Figure 4 shows that only the $R_{\mathrm{g}}$ data obtained by AFM agrees qualitatively with the $R_{\mathrm{h}}$ data, increasing monotonically with the drug concentration. The $R_{\mathrm{g}}$ data obtained by OT, on the other hand, increases until $C_{\mathrm{T}} / C_{\mathrm{bp}} \sim 1.9$ and then decreases, as a result from the abrupt decrease of the persistence length obtained in OT experiments (see Figure 2). As discussed, this behavior results from partial DNA melting due to the forces applied to perform the stretching experiments. ${ }^{[40]}$ Such forces are not present neither in AFM or DLS experiments, which explains the better agreement between these two techniques. In other words, the DLS results shown in Figure 4 agree with the partial melting assumption.

All these results together evidence that caution is needed when comparing persistence length data obtained from stretching and non-stretching techniques at least for DNAintercalators complexes. In fact one should expect that such techniques agree well only for low drug concentrations, since in this case the stretching forces used in the entropic regime $(F<5 \mathrm{pN})$ are not sufficient to induce partial melting on the DNA-intercalator complexes. ${ }^{[40]}$ Thus, for these types of complexes, the contour length data is more reliable to be used for extracting the physical chemistry of the interaction, as performed here.

\section{4 | CONCLUSIONS}

We have investigated the DNA interaction with the anticancer drug doxorubicin by using three very different experimental techniques: single molecule stretching performed by OT, single molecule imaging performed by AFM and ensemble-averaging DLS.

From the single molecule stretching experiments, we were able to determine the behavior of the basic mechanical parameters (persistence and contour lengths) as a function of the drug concentration in the sample.

The contour length data allowed us to extract the physical chemistry and to investigate the role of the ionic strength on the DNA-doxo binding. We have found that, at low ionic strengths, doxo interacts with DNA by simple intercalation (no aggregation) and/or by forming bound dimers. On the other hand, for high ionic strengths, doxo-doxo self-association is enhanced, giving rise to the formation of bound doxo aggregates composed by 3 to 4 molecules along the doublehelix.

The persistence length data obtained from single molecule stretching exhibits a non-monotonic behavior as a function of the doxo concentration in the sample, in contrast with the results obtained by single molecule imaging, which suggest a simple monotonic increase for this mechanical parameter. Such discrepancy was interpreted in terms of the external forces applied in the stretching experiments, which can partially melt the highly distorted double-helix of the DNA-intercalator complexes, resulting in a decrease of the effective persistence length.

Finally, we have performed DLS experiments in order to evaluate the changes on the effective size of the DNA-doxo complexes, represented here by the hydrodynamic radius, as a function of drug concentration. This parameter was qualitatively compared to the radius of gyration of the complexes, obtained both from OT and AFM, thus connecting the results obtained from the three techniques and corroborating with the partial melting proposal.

In summary, we have performed a robust characterization of the DNA-doxo complexes from the mechanical and physicochemical points of view, comparing results obtained from three very different experimental techniques. Such characterization has allowed an improvement in the understanding of the present interaction, revealing new peculiarities such as the dependence of the size of doxo aggregates on the buffer ionic strength, which allows one to modulate the physical chemistry of the interaction. In addition, the methods used here can be applied to other DNA binding ligands, thus 
providing clues about the mechanism(s) of action of important drugs.

\section{ACKNOWLEDGMENTS}

This work was supported by the Brazilian agencies: Fundação de Amparo à Pesquisa do Estado de Minas Gerais (FAPEMIG), Conselho Nacional de Desenvolvimento Científico e Tecnológico (CNPq) and Coordenação de Aperfeiçoamento de Pessoal de Nível Superior (CAPES). The DLS experiments were performed in "Laboratório de Microfluídica e Fluidos Complexos (LMFFC)" of Universidade Federal de Viçosa.

\section{REFERENCES}

[1] www.cancer.org, American Cancer Society 2014.

[2] D. Salerno, D. Brogioli, V. Cassina, D. Turchi, G. L. Beretta, D. Seruggia, R. Ziano, F. Zunino, F. Mantegazza, Nuc. Acids Res 2010, 38, 7089.

[3] C. Pérez-Arnaiz, N. Busto, J. M. Leal, B. García, J. Phys. Chem. B 2014, 118, 1288.

[4] M. Airoldi, G. Barone, G. Gennaro, A. M. Giuliani, M. Giustini, Biochem 2014, 53, 2197.

[5] D. Agudelo, P. Bourassa, G. Bérubé, H. Tajmir-Riahi, Intern. J. Biol. Macromol 2014, 66, 144.

[6] F. Yao, J. Duan, Y. Wang, Y. Zhang, Y. Guo, H. Guo, X. Kang, Anal. Chem 2015, 87, 338.

[7] J. R. Moffitt, Y. R. Chemla, S. B. Smith, C. Bustamante, Annu. Rev. Biochem 2008, 77, 205.

[8] I. de Vlaminck, C. Dekker, Annu. Rev. Biophys 2012, $41,453$.

[9] K. R. Chaurasiya, T. Paramanathan, M. J. McCauley, M. C. Williams, Phys. Life Rev 2010, 7, 299.

[10] F. Ritort, J. Phys. - Condens. Mat 2006, 18, R531.

[11] K. C. Neuman, A. Nagy, Nat. Methods 2008, 5, 491.

[12] I. Heller, T. P. Hoekstra, G. A. King, E. J. G. Peterman, G. J. L. Wuite, Chem. Rev 2014, 114, 3087.

[13] J. Zlatanovaa, S. M. Lindsayb, S. H. Leuba, Prog. Biophys. Mol. Biol 2000, 74, 61.

[14] I. Amitani, B. Liu, C. C. Dombrowski, R. J. Baskin, S. C. Kowalczykowski, Methods Enzymol 2010, 472, 261.

[15] K. Yoshikawa, S. Hirota, N. Makita, Y. Yoshikawa, Phys. Chem. Lett 2010, 1, 1763.

[16] J. Camunas-Soler, M. Manosas, S. Frutos, J. Tulla-Puche, F. Albericio, F. Ritort, Nucleic Acids Res 2015, 43, 2767.

[17] D. H. Paik, T. T. Perkins, Angew. Chem. Int. Ed 2012, $51,1811$.

[18] M. S. Rocha, Integr. Biol 2015, 7, 967.
[19] N. D. Fiori, A. Meller, Biophys. J 2010, 98, 2265.

[20] H. S. Chung, I. V. Gopich, K. McHale, T. Cellmer, J. M. Louis, W. A. Eaton, J. Phys. Chem. A 2011, 115, 3642.

[21] L. A. Reis, E. B. Ramos, M. S. Rocha, J. Phys. Chem. B 2013, 117, 14345.

[22] F. A. P. Crisafuli, E. B. Ramos, M. S. Rocha, Eur. Biophys. J 2015, 44, 1.

[23] J. B. Chaires, N. Dattagupta, D. M. Crothers, Biochemistry 1982, 21, 3927.

[24] M. Menozzi, L. Valentini, E. Vannini, F. Arcamone, J. Pharm. Sci 1984, 73, 766.

[25] J. F. Marko, E. D. Siggia, Macromolecules 1995, 28, 8759.

[26] F. A. P. Crisafuli, E. C. Cesconetto, E. B. Ramos, M. S. Rocha, Integr. Biol 2012, 2012, 568.

[27] E. C. Cesconetto, F. S. A. Junior, F. A. P. Crisafuli, O. N. Mesquita, E. B. Ramos, M. S. Rocha, Phys. Chem. Chem. Phys 2013, 15, 11070.

[28] C. Rivetti, M. Guthold, C. Bustamante, J. Mol. Biol 1996, 264, 919.

[29] J. S. Hur, E. S. G. Shaqfeh, J. Rheol 2001, 45, 421.

[30] L. Siman, I. S. S. Carrasco, J. K. L. da Silva, M. C. Oliveira, M. S. Rocha, O. N. Mesquita, Phys. Rev. Lett 2012, 109, 248103.

[31] E. F. Silva, E. B. Ramos, M. S. Rocha, J. Phys. Chem. B 2013, 117, 7292 .

[32] P. S. Alves, O. N. Mesquita, M. S. Rocha, J. Phys. Chem. Lett 2015, 6, 3549.

[33] M. S. Rocha, M. C. Ferreira, O. N. Mesquita, J. Chem. Phys 2007, 127, 105108.

[34] M. S. Rocha, A. D. Lúcio, S. S. Alexandre, R. W. Nunes, O. N. Mesquita, Appl. Phys. Lett 2009, 95, 253703.

[35] J. E. Coury, L. McFail-Isom, L. D. Williams, L. A. Bottomley, P. Natl. Acad. Sci. USA 1996, 93, 12283.

[36] J. D. McGhee, P. H. von Hippel, J. Mol. Biol 1974, 86, 469.

[37] J. B. Chaires, N. Dattagupta, D. M. Crothers, Biochemistry 1982, 21, 3933.

[38] J. Camunas-Soler, S. Frutos, C. V. Bizarro, S. de Lorenzo, M. E. Fuentes-Perez, R. Ramsch, S. Vilchez, C. Solans, F. Moreno-Herrero, F. Albericio, R. Eritja, E. Giralt, S. B. Dev, F. Ritort, ACS Nano 2013, 7, 5102.

[39] X. M. Hou, X. H. Zhang, K. J. Wei, C. Ji, S. X. Dou, W. C. Wang, M. Li, P. Y. Wang, Nucleic Acids Res 2009, 37, 1400 .

[40] R. F. Bazoni, C. H. M. Lima, E. B. Ramos, M. S. Rocha, Soft Matt 2015, 11, 4306.

[41] T. Hwa, E. Marinari, K. Sneppen, L. H. Tang, Proc. Natl. Acad. Sci. USA 2003, 100, 4411. 
[42] A. K. Dasanna, N. Destainville, J. Palmeri, M. Manghi, 1, Phys. Rev. E 2013, 87, 052703.

[43] F. Sicard, N. Destainville, M. Manghi, J. Chem. Phys 2015, 142, 034903.

[44] . D. Vladescu, M. J. McCauley, M. E. Nunez, I. Rouzina, M. C. Williams, Nat. Methods 2007, 4, 517.

[45] M. Daune, Molecular Biophysics, 1st ed.; Oxford University Press: Oxford, 1999.

Reviewing Editor: Kenneth Breslauer

\section{SUPPORTING INFORMATION}

Additional Supporting Information may be found in the online version of this article.

How to cite this article: Silva EF, Bazoni RF, Ramos $\mathrm{EB}$, and Rocha MS. DNA-doxorubicin interaction: New insights and peculiarities. Biopolymers. 2017;107: e22998. doi:10.1002/bip.22998. 